Journal of Computer Science 8 (5): 747-751, 2012

ISSN 1549-3636

(C) 2012 Science Publications

\title{
Medical Image Compression with Lossless Region of Interest Using Adaptive Active Contour
}

\author{
${ }^{1}$ Loganathan, R. and ${ }^{2}$ Y.S. Kumaraswamy \\ ${ }^{1}$ Department of Computer Science and Engineering, \\ Sathyabama University, Chennai, 600 119, Tamil Nadu, India \\ ${ }^{2}$ Department of Computer Science and Engineering, \\ Department of MCA (VTU), Dayanada Sagar College of Engineering, \\ Kumarawsamy Layout, Bangalore, 560078, Karnataka, India
}

\begin{abstract}
Problem statement: With increasing bandwidth, digital medical image storage and transmission is a boon to patients and health professionals alike. Medical images are available instantly and avoid the need to carry the data physically. Popular imaging techniques extensively used in medicine include X-Ray, Magnetic Resonance Imaging (MRI), Ultrasound and Computed Tomography (CT). The images produced from the above techniques can be segregated into spatial regions with some regions more important for diagnosis compared to other regions. The region of interest for diagnosis is usually a small area compared to the whole image captured. Compression techniques play a very important role for fast and efficient transfer of medical images. Lossless compression techniques ensure no data loss but have the limitations of low compression rate. Lossy compression techniques on the other hand provide better compression ratios but the cost of wrong diagnosis is very high. In this study it is proposed to explore multiple compression techniques based on Region OF Interest (ROI). Approach: In this study a novel active contour method is proposed which is adaptive and marks the outer region of interest without edges. Based on the ROI, the active area of interest is compressed using lossless compression and the other areas compressed with lossy wavelet compression techniques. Results and Conclusion: Our proposed procedure was applied to different MRI images obtaining overall compression ratios of $70-80 \%$ without losing the originality in the ROI.
\end{abstract}

Key words: Region OF Interest (ROI), Computed Tomography (CT), Magnetic Resonance Imaging (MRI), network connectivity, Mean Square Error (MSE), Discrete Wavelet Transform (DWT), Peak Signal to Noise Ratio (PSNR), efficient transfer

\section{INTRODUCTION}

Image compression is a necessity for most telematic applications and plays a crucial role to ensure good quality of service (Assche et al., 1999). It is necessary that medical images be transmitted fast with high reliability so that medical diagnosis at remote locations with poor network connectivity can be easily facilitated. To this end, image compression plays an important role to reduce the bandwidth required during the connection. The challenge however is that while high compression rates are desired, the usability of the reconstructed images depends on certain significant characteristics of the original images which need to be preserved after the compression process has been finished (Bullmore et al., 2004).

Image compression reduces the amount of data required to represent an image with close resemblance to the original image by removing redundant information. Three types of redundancies for digital images are generally exploited by compression algorithms. These are, coding redundancy that arises from the representation of the image gray levels, inter pixel redundancy as there is a high similarity between neighboring pixels in a major percentage of the image and visual redundancy that is based on Human perception of the image information (Fowler and Pesuet-Popescu, 2007). An image compression system consists of an encoder that exploits the redundancies to represent the image data in a compressed manner. Whereas the decoder is used to reconstruct the original image from the compressed data (Ghrare et al., 2009).

Compression algorithms for image compression can either be lossless or lossy. Images compressed in a lossless manner can be reconstructed without any

Corresponding Author: Loganathan, R., Department of Computer Science and Engineering, Sathyabama University, 
change in the pixel intensity which limits the amount of compression. However, many applications such as satellite image processing and certain medical an document imaging, cannot afford any losses in their data and are normally compressed using lossless compression methods (Al-Azawi et al., 2012). Lossy encoding methods are based on trading off achieved in either compression or bit rate with the distortion of the reconstructed image. Lossy encoding for images is usually obtained using transforms which can be converting data from spatial domain to frequency domain. Transform domain removes the redundancies by mapping the pixels into the transform domain before encoding. This results only in a few transform coefficients. For compression, only the few significant coefficients need be encoded, while a majority of the insignificant coefficients can be removed without significantly affecting the quality of the reconstructed image. An ideal transform mapping should be reversible and able to completely decor relate the transform coefficients.

Compressing an image is significantly different than compressing raw binary data (Goldberg et al., 1994). General purpose compression programs can also be used to compress images, but the result obtained are typically less than optimal as images have certain statistical properties which can be exploited by encoders specifically designed for them. Two of the error metrics used to compare the various image compression techniques are the Mean Square Error (MSE) and the Peak Signal to Noise Ratio (PSNR) (Muthaiah et al., 2008). The MSE is the cumulative squared error between the compressed and the original image, whereas PSNR is a measure of the peak error. The mathematical formulae for the two are:

$$
\operatorname{MSE}=\frac{1}{\mathrm{MN}} \sum_{\mathrm{y}=1}^{\mathrm{M}} \sum_{\mathrm{x}=1}^{\mathrm{N}}\left[1(\mathrm{x}, \mathrm{y})-1^{\prime}(\mathrm{x}, \mathrm{y})\right]^{2}
$$

And:

$$
\mathrm{PSNR}=20 * \log 10(255 / \mathrm{sqrt}(\mathrm{MSE}))
$$

where, $I(x, y)$ is the original image, $I^{\prime}(x, y)$ is the roximated version (which is actually the decompressed image) and M,N are the dimensions of the images. A lower value for MSE means lower error and as seen from the inverse relation between the MSE and PSNR, which translates to a high value of PSNR. Logically, a higher value of PSNR is good because it means that the ratio of Signal to Noise is higher. Here, the 'signal' is the original image and the 'noise' is the error in reconstruction.
An image can be represented as a two-dimensional array of coefficients, each coefficient representing the brightness level of the pixel. Looking from a general perspective, we can't differentiate between coefficients which are important and which are not. Most natural images have smooth colour variations, with the fine details being represented as sharp edges in between the smooth variations. The smooth variations in colour can be termed as low frequency variations and the sharp variations as high frequency variations which can be a prime source for encoding algorithms.

The low frequency components constitute the base of an image and the high frequency components add upon them to refine the image, thereby giving a detailed image. Separating the smooth variations and details of the image can be achieved using a Discrete Wavelet Transform (DWT). In DWT a low pass filter and a high pass filter are chosen, such that they exactly halve the frequency range between themselves. This filter pair is called the Analysis Filter pair. The process starts by applying the low pass filter for each row of data, there by getting the low frequency components of the row (Gonzalez and Richard, 2002). But since the low pass filter is a half band filter, the output data contains frequencies only in the firsthalf of the original frequency range, thus by Shannon's Sampling Theorem, they can be subsampled by two, so that the output data now contains only half the original number of samples. Next, the high pass filter is applied for the same row of data and similarly the high pass components are separated and placed by the side of the low pass components. This procedure is done for all rows. The reverse is applied to reconstruct the image. For reconstruction the filter pair is called the Synthesis Filter pair. The filtering procedure is exactly the opposite of the decomposition method.

Wavelets are created by dilating and translating a single prototype function or wavelet $\psi(\mathrm{t})$ :

$$
\psi \mathrm{a}, \mathrm{b}(\mathrm{t})=|\mathrm{a}|^{-1 / 2} \psi\left(\frac{\mathrm{t}-\mathrm{b}}{\mathrm{a}}\right)
$$

The mother or basic wavelet [10] $\psi(\mathrm{t})$ must satisfy

$\int \psi(x) \mathrm{d} x=0$.

The continuous wavelet transform of $f(t)$ with respect to the wavelet $\psi(\mathrm{t})$ can be given by:

$\mathrm{W}_{\mathrm{f}}(\mathrm{a}, \mathrm{b})=<\mathrm{f}(\mathrm{t}), \psi_{\mathrm{a}, \mathrm{b}}>=\int_{-\infty}^{\infty} \mathrm{f}(\mathrm{t}) \psi_{\mathrm{a}, \mathrm{b}}(\mathrm{t}) \mathrm{dt}$

The inverse of the continuous wavelet transform is given by: 


$$
f(t)=\frac{1}{C \psi} \int_{0}^{\infty} \int_{-\infty}^{\infty} W_{f}(a, b) \Psi_{a, b}(t) d b \frac{d a}{a^{2}}
$$

Another type of wavelet is based on the binary scaling and dyadic translations to form the basis functions. For a signal $\mathrm{f}(\mathrm{t})$ the output becomes:

$$
f(t)=\sum_{k} \sum_{j} a_{j, k} \psi_{j, k}(t)
$$

$\psi_{\mathrm{j}, \mathrm{k}}(\mathrm{t})$ formed from the mother wavelet $\psi(\mathrm{t})$ produce the wavelet expansion functions that can form an orthogonal basis defined by:

$$
\psi_{\mathrm{j}, \mathrm{k}}(\mathrm{t})=2^{\mathrm{j} / 2} \psi\left(2^{\mathrm{j}} \mathrm{t}-\mathrm{k}\right)
$$

where, $\mathrm{j}$ determines the dilation and the translation is specified by $\mathrm{k}$. The two dimensional value is also known as discrete wavelet transform.

The biorthogonal wavelets introduced by Cohen, Daubechies and Feauveau contain in particular compactly supported biorthogonal spline wavelets compactly supported duals. In biorthogonal wavelets, separate decomposition and reconstruction filters are defined and hence the responsibilities of analysis and synthesis are assigned to two different functions (in the biorthogonal case) as opposed to a single function in the orthonormal case (Grgic et al., 2001; Ho et al., 1993; Shapiro, 1993).

The biorthogonal scaling function is given by:

$$
\begin{aligned}
& \phi(t)=2 \sum_{n-\infty}^{\infty} \mathrm{h}(\mathrm{n}) \phi(2 \mathrm{t}-\mathrm{n}) \operatorname{dual} \tilde{\phi}(\mathrm{t})=2 \sum_{\mathrm{n}-\infty}^{\infty} \tilde{\mathrm{h}}(\mathrm{n}) \tilde{\phi}(2 \mathrm{t}-\mathrm{n}) \\
& \langle(\mathrm{t}),), \tilde{\phi}(\mathrm{t}-\mathrm{k})\rangle=\delta(\mathrm{k})\left\langle\phi\left(2^{-\mathrm{k}} \mathrm{t}\right), \tilde{\phi}\left(2^{-\mathrm{k}} \mathrm{t}-\mathrm{n}\right)\right\rangle=2^{\mathrm{k}} \delta(\mathrm{n})
\end{aligned}
$$

where, $h(n)$ and $\tilde{h}(n)$ serve as impulse response of FIR filters and two sets of scaling functions $\psi(\mathrm{t})$ and $\tilde{\phi}(\mathrm{n})$ generate subspaces $\mathrm{V}_{\mathrm{k}}$ and $\widetilde{\mathrm{V}}_{\mathrm{k}}$ respectively. Unlike the orthogonal case, it is possible to synthesize biorthogonal wavelets and scaling functions which are symmetric or antisymmetric and compactly supported.

Though various compression ratios with maximum energy retained have been proposed in literature, improving the compression ratio while retaining the maximum energy hits the road block as medical images are highly sensitive to noise. To overcome the limitations of lossy compression different compression techniques on the same image based on the area of interest has been proposed.

\section{MATERIALS AND METHODS}

Initial approach to segment an image were based on edge detection. Canny edge detection and Sobel edge detection has been popular and extensively used in literature. For medical images in Fig. 1 the edge detection by Sobel and Canny is shown in Fig. 2 and 3. For ROI based compression it is important to distinguish the ROI and the non ROI regions. From the figures it is observed that edge detection methods try to show the edges inside the ROI.

CHAN-VESE active contour algorithm comes from segmentation problem formulated by Mumford and Shah Active contour model, also called snakes, is a framework for delineating an object outline from a possibly noisy $2 \mathrm{D}$ image. This framework attempts to minimize an energy associated to the current contour as a sum of an internal and external energy:

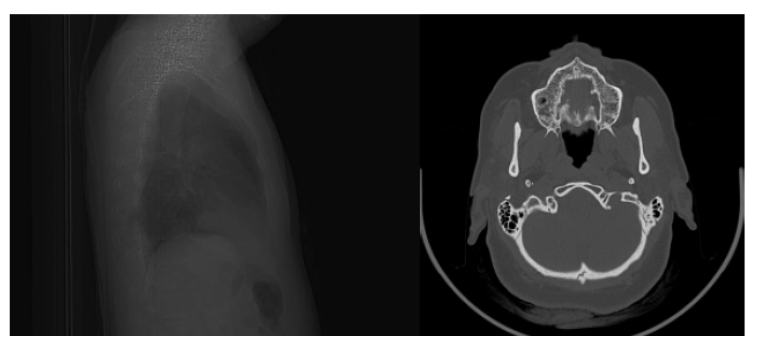

Fig. 1: Original Image

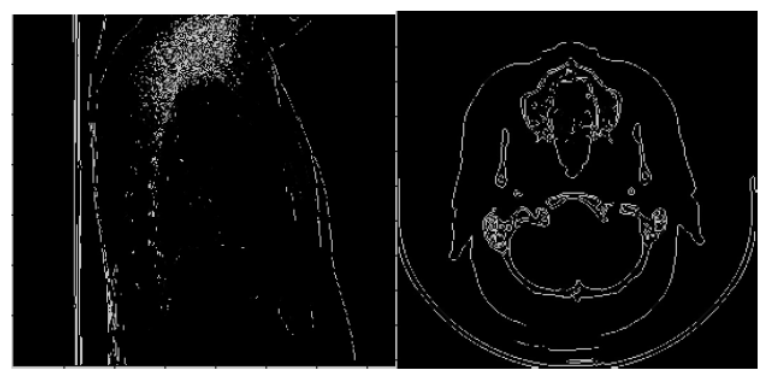

Fig. 2: Sobel edge detection

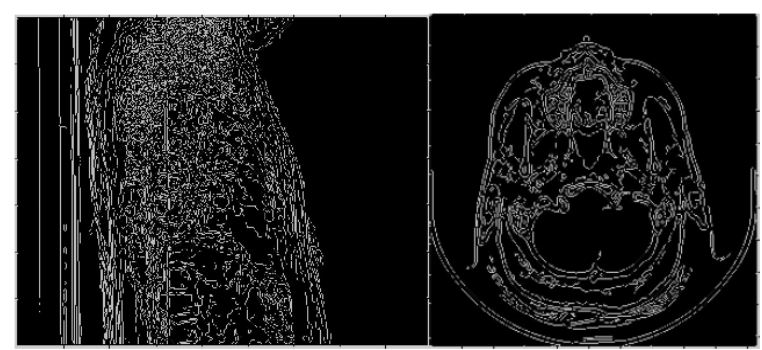

Fig. 3: Canny edge detector 
- The external energy is supposed to be minimal when the snake is at the object boundary position. The most straightforward approach consists in giving low values when the regularized gradient around the contour position reaches its peak value

- The internal energy is supposed to be minimal when the snake has a shape which is supposed to be relevant considering the shape of the sought object. The most straightforward approach grants high energy to elongated contours (elastic force) and to bended/high curvature contours (rigid force), considering the shape should be as regular and smooth as possible

The basic Active model is given by:

$$
\begin{aligned}
& \mathrm{F}\left(\mathrm{c}^{+} \mathrm{c}^{-}, \mathrm{C}\right)=\mu \text {. Length }(\mathrm{C})+\lambda+\int_{\text {niside(C) }}\left|\mu 0(\mathrm{x}, \mathrm{y})-\mathrm{c}^{+}\right|^{2} \mathrm{~d} \times \mathrm{dy} \\
& +\lambda-\int_{\text {outsidec }(\mathrm{C})}\left|\mu 0(\mathrm{x}, \mathrm{y})-\mathrm{c}^{-}\right|^{2} \mathrm{~d} \times \mathrm{dy}
\end{aligned}
$$

where, $\mathrm{c}+$ and $\mathrm{c}-$ are constant unknowns representing the average value of $\mathrm{u} 0$ inside and outside the curve, respectively.

The parameters $\mu>0$ and $\lambda_{+}, \lambda_{-}>0$ are weights for the regularizing term and the fitting term, respectively. The above model can be rewritten as:

$$
\begin{aligned}
& \mathrm{F}\left(\mathrm{c}^{+} \mathrm{c}^{-}, \phi\right)=\mu . \operatorname{Length}\{\phi=0\}+\lambda+\int_{\phi \dot{\phi} 0}\left|\mu 0(\mathrm{x}, \mathrm{y})-\mathrm{c}^{+}\right|^{2} \mathrm{dxdy} \\
& +\lambda-\int_{\phi<0}|\mu 0(\mathrm{x}, \mathrm{y})-\mathrm{c}-|^{2} \mathrm{dxdy}
\end{aligned}
$$

For the level set formation where

Chen Vese Active contour uses the Heaviside function and the dirac delta function. To avoid the hard and:

$$
\mathrm{C}=\{(\mathrm{x}, \mathrm{y}) \in \Omega: \phi(\mathrm{x}, \mathrm{y})=0\}
$$

Sensitive boundary, we introduce the fuzzy Heaviside model. In the level set we introduce a fuzzy function with membership values given by:

$\mathrm{H}(\mathrm{z})=1$ when $\mathrm{z}$ between $0.9-1.1$

$\mathrm{H}(\mathrm{z})=0$ when $\mathrm{z}$ between 0.1 and negative

Number and the Dirac delta function:

$$
\delta(Z)=\frac{d}{d z} H(z)
$$

We can rewrite the energy function a follows:

$$
\begin{aligned}
& \mathrm{F}\left(\mathrm{c}^{+}, \mathrm{c}^{-}, \phi\right)=\mu \int_{\Omega} \delta\left(\phi(\mathrm{x}, \mathrm{y}) \nabla \phi(\mathrm{x}, \mathrm{y})+\lambda^{+}\right. \\
& \int_{\Omega}\left|\mu 0(\mathrm{x}, \mathrm{y})-\mathrm{c}^{+}\right|^{2} \mathrm{H}(\phi(\mathrm{x}, \mathrm{y})) \mathrm{dxdy} \\
& \left.+\lambda^{-} \int_{\Omega}\left|\mu_{0}(\mathrm{x}, \mathrm{y})-\mathrm{c}^{-}\right|^{2}(1-\mathrm{H})(\phi(\mathrm{x}, \mathrm{y}))\right) \mathrm{dxdy}
\end{aligned}
$$
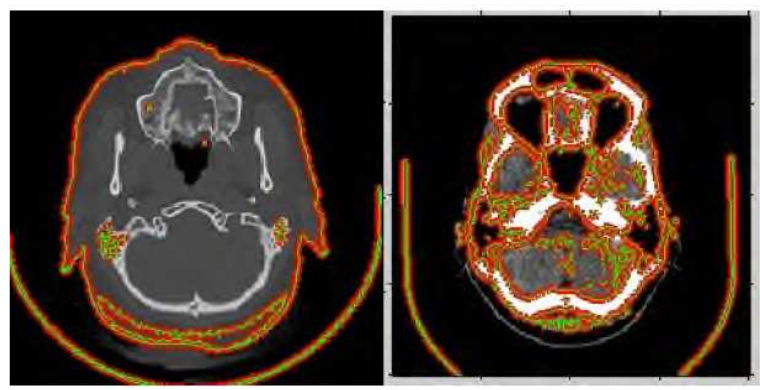

Fig. 4: The edges detected using proposed method
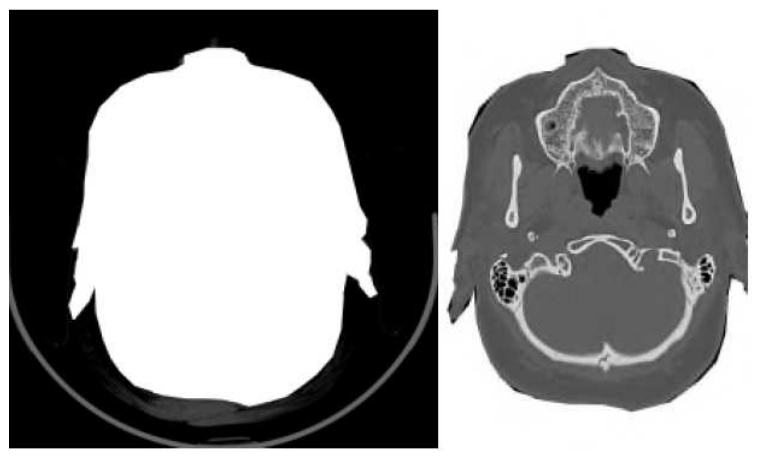

Fig. 5: Extracted image

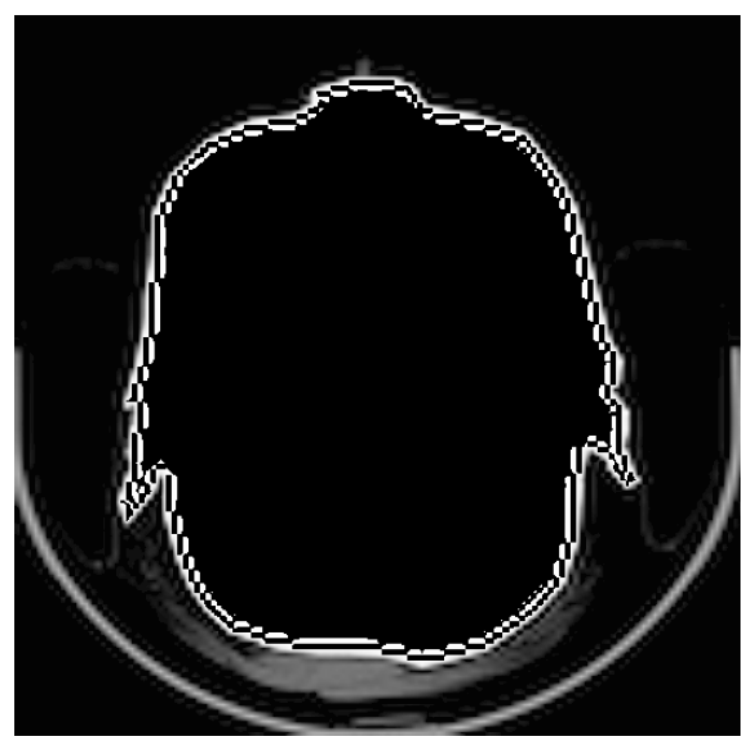

Fig. 6: Highly compressed non region of interest 
Table 1: Compression ratios for various images

\begin{tabular}{lll}
\hline $\begin{array}{l}\text { Name of } \\
\text { image }\end{array}$ & $\begin{array}{l}\text { Compression } \\
\text { technique }\end{array}$ & $\begin{array}{l}\text { Compression } \\
\text { ratio (\%) }\end{array}$ \\
\hline Original Image & Bior 2.6 Lossless & 31.37 \\
ROI of original image & Bior 2.6 Lossless & 59.90 \\
Non ROI part of original image & Bior 2.6 Lossy & 93.32 \\
\hline
\end{tabular}

The proposed model was implemented in Matlab. Figure 4 shows the input and output for different medical images. The outer edge becomes the mask to compute the ROI between the important space and the non important space. Figure 5 shows the extracted ROI and Non ROI part of the image.

Lossy compression using $\mathrm{Bi}$ orthorgornal spline wavelet with $\mathrm{N}_{\mathrm{r}}=-2$ and $\mathrm{N}_{\mathrm{d}}=6$ was performed on the Non ROI part and lossless compression using the same technique for original and ROI part of the image. Figure 6 shows the Non ROI image after lossy compression.

\section{RESULTS AND DISCUSSION}

Using the proposed method for segregating ROI and non ROI, it is seen that the overall compression ration improves to a large extent as shown in Table 1.

Assuming that the Non ROI covers $60 \%$ of the medical image then the total compression that could be achieved could be in the region of $70-80 \%$. This is compared to lossless compression technique on the whole image which produces compression ratios in the range of $30-40 \%$. The originality of the image is not lost and the health professional does not lose the originality of the captured image.

\section{CONCLUSION}

In this study it was proposed to describe an image segmentation process based on Region Of Interest (ROI) for lossless compression on the ROI and lossy compression for the non ROI. This method of compression finds extensive application in medical images where the originality of the image should be retained. An improved active contour technique to find the region of interest based on fuzzy logic was proposed. The proposed method provided better compression method compared to lossless compression on the whole image

\section{REFERENCES}

Assche, S.V., D.D.E. Rycke, W. Philips and I. Lemahieu, 1999. Exploiting interframe redundancies in the lossless compression of 3D medical images. University of Gent, Belgium.
Al-Azawi, S., S. Boussakta and A. Yakovlev, 2012. Image compression algorithms using intensity based adaptive quantization coding. Am. J. Eng. Applied Sci., 4: 504-512. DOI: 10.3844/ajeassp.2011.504.512

Bullmore, E., J. Fadili, V. Maxim, L. Sendur and J. Suckling et al., 2004. Wavelets and functional magnetic resonance imaging of the human brain. NeuroImage, 23: 234-249. DOI: 10.1016/j.neuroimage.2004.07.012

Fowler, J.E and B. Pesuet-Popescu, 2007. An Overview on Wavelets in Source Coding, Communications and Networks. EURASIP J. Image Video Process. DOI: $10.1155 / 2007 / 60539$

Ghrare, S.E.M., A.M. Ali, K. Jumari and M. Ismail, 2009. An efficient low complexity lossless coding algorithm for medical images. Am. J. Applied Sci., 6: $\quad 1502-1508 . \quad$ DOI: 10.3844/ajassp.2009.1502.1508

Goldberg, M.A., M. Pivovarov, WW. Mayo-Smith, MP. Bhalla and JG. Blickman et al., 1994. Application of wavelet compression to digitized radiographs. AJR.,163: 463-468. PMID: 8037051

Gonzalez, R.C. and E. Richard, 2002. Woods. Digital Image Processing. Pearson Education, Englewood Cliffs, ISBN-10: 0201180758, pp: 793.

Grgic, S., M. Grgic and B. Zorkocihlar, 2001. Performance Analysis of image compression using wavelets. IEEE Trans. Ind. Electr., 48: 682-695. DOI: $10.1109 / 41.925596$

Ho, B.K.T., V. Tseng, M. Ma and D. Chen, 1993. A mathematical model to quantify JPEG block artifacts. Proc. SPIE., pp: 269-274. DOI: 10.1117/12.146974

Muthaiah, R., K. NeelaKantan, V. Sharma and A. Arora, 2008. Image Compression and Reconstruction using Cubic Spline Interpolation Technique. Am. J. Applied Sci., 5: 1562-1565.

Shapiro, J.M., 1993. Embedded image coding using zerotrees of wavelet coefficients. IEEE Trans. Signal Process., 41: 3445-3462. DOI: 10.1109/78.258085 